\title{
The power of words: sources of anxiety in patients undergoing local anaesthetic plastic surgery
}

\author{
J Caddick $^{1}$, S Jawad ${ }^{2}$, S Southern ${ }^{1}$, S Majumder ${ }^{1}$ \\ ${ }^{1}$ Mid Yorkshire Hospitals NHS Trust, UK \\ ${ }^{2}$ Leeds Partnerships NHS Foundation Trust, UK
}

ABSTRACT

INTRODUCTION With local or regional anaesthesia being employed for more as well as more complex surgical procedures, an increasing number of patients remain fully conscious during their operation. This is generally perceived as being advantageous to the patient as less time is spent in hospital and the side effects of general anaesthesia are avoided. However, there is no direct measure of the patient experience during 'awake surgery', in particular of which aspects of the process may be distressing. METHODS Seventy patients undergoing day case plastic surgery under local anaesthesia were asked to complete a short questionnaire immediately following their operation. This was designed to identify specific factors likely to either increase or reduce anxiety during surgery. The questionnaire was initially validated on a pilot group of ten patients.

RESULTS Unsurprisingly, painful stimuli such as injections were identified as potential stressors. More interestingly, the data highlighted that some commonly used surgical terms such as 'knife' and 'scalpel' provoke considerable anxiety in the conscious patient. This varied according to age and sex with younger and female patients being most vulnerable. Other events identified as potential stressors, such as casual conversations and movements among theatre staff, were actually shown to be non-stressful and, in some cases, stress relieving.

CONCLUSIONS Technical jargon used by surgical staff can elevate anxiety levels among patients who are awake for their operation. Careful consideration of the words we use may reduce this, particularly in female patients.

\section{KEYWORDS}

Local anaesthesia - Anxiety - Communication - Surgical jargon

Accepted 30 October 2011

\section{CORRESPONDENCE TO}

Jenny Caddick, SpR in Plastic Surgery, Pinderfields Hospital, Aberford Road, Wakefield, West Yorkshire WF1 4DG, UK

E: jennycaddick@doctors.org.uk

Technological and pharmacological developments and the expansion of day case surgery in modern surgical practice have resulted in increased use of local anaesthetic techniques. ${ }^{1}$ As more complex procedures are performed under local or regional anaesthesia, an increasing number of patients remain fully conscious during their operation. Studies examining a broad range of surgical procedures suggest that having a procedure performed under local or regional rather than general anaesthesia is generally advantageous to the patient with reports of shorter hospital stays, reduced need for opiate analgesia post-operatively, lower complication rates, increased patient satisfaction and even a reduction in cancer recurrence. ${ }^{2-6}$ In addition, costs to the hospital may be reduced.

Nevertheless, there is a paucity of information regarding the overall patient experience during 'awake surgery'. While an explanation of the type of anaesthesia and potential surgical complications is standard practice, specific information about the theatre environment can be left very much to the imagination. Despite this, we expect our patients to walk, often semi-clothed, into a room of strangers, lie motionless on a narrow table and, during their procedure, be subjected to both detailed technical discussion and casual conversations among theatre staff.

There is little evidence in the available literature regarding which, if any, aspects of this process may be distressing. Anecdotally, however, among our own plastic surgery patients, certain aspects of the intra-operative experience have been identified as stressful. Examples of these include the surgical terminology used, movement and activity in the theatre and casual conversations among theatre staff.

It has been suggested that increased patient anxiety in the peri-operative period impacts detrimentally on both intra-operative risk ${ }^{8}$ and post-operative recovery. ${ }^{9}$ We therefore postulate that an attempt to identify and then reduce potentially stressful aspects of the operative process would have a positive impact on our patients, both psychologically and in terms of physical recovery. In this study we have attempted to identify which aspects of the local anaesthetic process cause distress in our patients attending for minor, day case plastic surgery. 


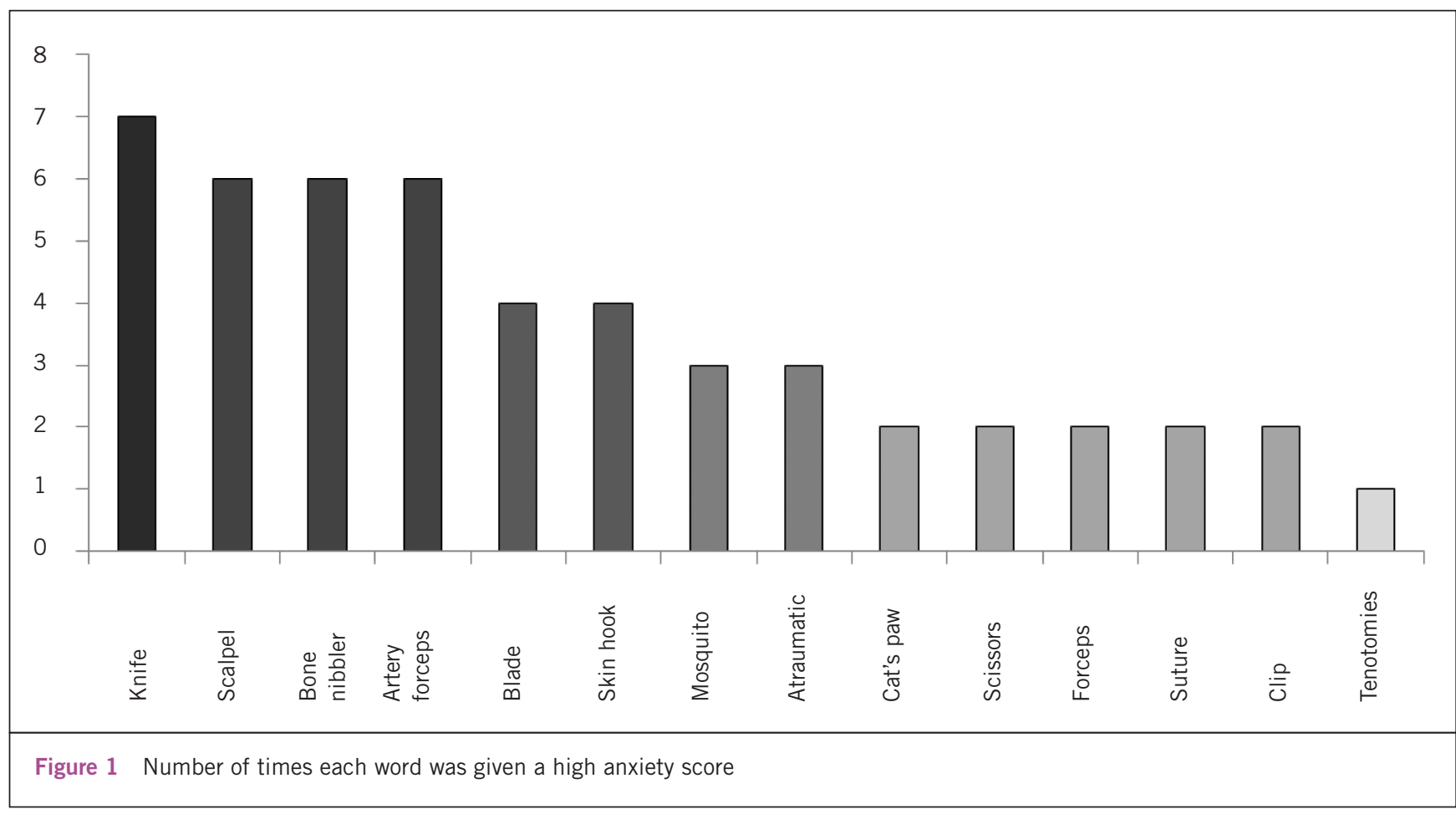

Table 1 Level of anxiety provoked by surgical terms

\begin{tabular}{|llllll|}
\hline & $\begin{array}{l}\mathbf{1} \\
\text { (no } \\
\text { anxiety) }\end{array}$ & $\mathbf{2}$ & $\mathbf{3}$ & $\mathbf{4}$ & $\begin{array}{l}\mathbf{5} \\
\text { (very } \\
\text { anxious) }\end{array}$ \\
\hline Knife & 45 & 9 & 4 & 1 & 6 \\
Blade & 46 & 9 & 3 & 1 & 3 \\
Scalpel & 43 & 11 & 4 & 2 & 4 \\
Scissors & 51 & 8 & 1 & 1 & 1 \\
\hline Tenotomies & 52 & 5 & 2 & 1 & 0 \\
Skin hook & 44 & 8 & 6 & 3 & 1 \\
Cat's paw & 51 & 6 & 3 & 2 & 0 \\
Forceps & 51 & 6 & 2 & 1 & 1 \\
Bone & 45 & 6 & 4 & 4 & 2 \\
nibbler & & & & & \\
Suture & 54 & 5 & 3 & 1 & 1 \\
Atraumatic & 48 & 5 & 5 & 2 & 1 \\
Clip & 56 & 3 & 1 & 2 & 0 \\
Mosquito & 53 & 3 & 2 & 2 & 1 \\
Artery & 45 & 4 & 6 & 3 & 3 \\
forceps & & & & & \\
\hline
\end{tabular}

\section{Methods}

Seventy-three patients undergoing elective, day case, local anaesthetic, non-cosmetic plastic surgery during August 2010 were included in this study. Three were later excluded due to incompletely filled in questionnaires. All patients underwent routine pre-operative counselling on the day of admission. The surgeons obtaining consent from the patients were not aware that the study was being carried out at that time although all had previously agreed to their patients being entered into the study. The study was approved by the local ethics committee. All responses were anonymous and participants were informed that their withdrawal from the study or a decision not to participate would not affect their care.

Patients were asked to complete a short questionnaire within 30 minutes of surgery. The questionnaire was initially validated on a pilot group of 10 patients. It used a combination of direct, closed response answers, free text and a 5-point numerical rating (Likert-type) scale to measure anxiety where 1 represented 'no anxiety' and 5 'very anxious'. This scale was applied to potential stressors identified previously from anecdotal comments made by patients undergoing local anaesthetic procedures. Basic demographics (age and sex) were also collected.

The procedures performed represent a routine range of day case, elective, minor plastic surgery cases. Thirty-seven per cent of operations were on the upper limb, all of which were performed under tourniquet control. The remaining $63 \%$ were principally excision of possible skin malignancies. Two-thirds $(61 \%)$ of these involved the head and neck region and $32 \%$ involved some kind of reconstruction (either a local flap or a skin graft).

\section{Results}

Of the 70 patients who completed the questionnaire, 43 (61\%) were women and 27 (39\%) were men. The age range was 16-91 years with a median age of 61 .

Only one patient stated that the general theatre environment made him or her anxious. A greater proportion (10\%) 
identified aspects of the operation itself as causing distress. The most commonly recorded source of distress was the injection of local anaesthesic (6\% of the total group). Other factors commented on were the use of a tourniquet ( $4 \%$ of patients undergoing hand surgery) and the distress caused by surgeons operating around the face $(7 \%$ of patients undergoing removal of head and neck lesions).

Patients graded commonly used surgical terms on a fivepoint scale to identify words that were more likely to cause anxiety (Table 1). 'Knife' was rated as most alarming, closely followed by 'scalpel', 'bone nibbler' and 'artery forceps' (Fig 1). In some cases, where more than one descriptive term for a surgical tool exists, the results indicate that use of a well recognised alternative may cause less anxiety. Only $6 \%$ of patients scored the word 'blade' as causing significant anxiety compared with $11 \%$ for the word 'knife'. Conversely, the term 'artery forceps' was identified as stressful by $10 \%$ of patients while the commonly used but slightly more ambiguous alternative 'clip' only provoked anxiety in $2 \%$.

When the results were broken down according to patient age, there was no consistent age related pattern. The lowest mean number of high scores per person was 0.3 in the 45-65 age group and the highest was 1.23 in the $25-45$ year age group.

Considering the sex of the patient, women were far more likely to give higher anxiety scores related to surgical instruments than their male counterparts, comprising $76 \%$ of the total number of high scores given. This held true when allowance was given for the larger proportion of women in the cohort. Female patients gave an average of 0.91 high scores per person and men less than half of that (0.44).

In addition to specific questions relating to surgical instruments, patients were also asked to score the stress inducing effects of background noise. In our theatre environment this relates principally to staff movements in the operating theatre, casual conversations among theatre staff and low volume radio music. The results indicate that none of these factors had an appreciable impact on patient anxiety with less that $2 \%$ of patients recording high anxiety scores. Furthermore, a small proportion of patients (3\%) commented that listening to staff banter in theatre was actually comforting.

\section{Discussion}

Anxiety, an unpleasant state of unease or tension, is experienced frequently by the pre-operative patient. ${ }^{10}$ Not only an unpleasant experience, anxiety can influence the volume of anaesthetic required, the safe maintenance of the patient intra-operatively and the success of the surgical procedure itself. ${ }^{8,10,11}$ This, in turn, impacts on post-operative recovery and the development of emotional/anxiety states. ${ }^{8}$ It is therefore in the interest of all surgeons to identify the precipitants of intra-operative anxiety in order to reduce their well documented negative impact.

Unsurprisingly, our study identified that factors causing pain, such as the injection of local anaesthetic and the application of an upper limb tourniquet, are most likely to precipitate anxiety. However, the numbers in both cases were small. Simple measures such as warming the local anaes- thetic ${ }^{12}$ and using the smallest available needle guage ${ }^{13}$ have been well described and should always be used where possible to reduce discomfort.

The second most common cause of distress related to touching or covering over of the face. This experience is not limited to plastic surgical patients and has been documented previously in those undergoing ophthalmological surgery. The distress relates principally to a perception that surgery on the face impairs communication. ${ }^{14,15}$ Techniques that offer a clear alternative method of communication such as hand holding or a handheld buzzer can alleviate this anxiety. ${ }^{16}$

The importance of direct communication and information provision in the alleviation of peri-operative anxiety is well documented. ${ }^{15,17-19}$ However, for patients undergoing conscious surgery, further, unwanted information may be communicated to them inadvertently in the form of both technical and casual conversations between staff. When facing surgery, patients exhibit heightened and focused attention as well as increased susceptibility to suggestion. In this state, negative suggestion, usually words spoken unintentionally, can aggravate anxiety, stress and pain..$^{20}$

There is no information in the available literature regarding the effect of the specific surgical words used in theatre. However, patients awaiting minor gynaecological procedures experienced higher levels of anxiety when they were exposed to physically threatening words compared with neutral words. ${ }^{21}$ Further studies have noted an increase in patient anxiety at the sight ${ }^{22}$ or sound of surgical instruments. ${ }^{25}$ Fear of hearing or seeing aspects of their procedure was a common reason cited by patients who opted for general over regional anaesthesia for a Caesarean section. ${ }^{24}$

In this study, the first of its kind to do so, we have been able to identify individual, commonly used surgical terms that patients report as anxiety provoking. This included words that are in common general usage (knife) and those less familiar to the lay person but used by many surgical specialties (eg artery forceps and bone nibbler). The results allowed us to rank these surgical terms in order of how threatening they are perceived to be. Thus, the word 'knife' is ranked as more alarming than 'blade' and 'artery forceps' as more alarming than 'clip'. This suggests that careful selection of the term used could have a positive impact on anxiety levels in the conscious patient.

Interestingly, our findings show a dramatic sex imbalance. This finding is supported by studies in other surgical specialties that have found overwhelmingly that female patients undergoing surgery experience higher levels of anxiety. ${ }^{25-27}$ What is less clear is why this imbalance exists. Are male patients tougher than their female counterparts or less willing to report anxiety? Or are our female patients listening more closely to the discussions going on in theatre? The literature suggests that female surgical patients have a significantly higher need for information than $m^{28}$ and that it is satisfaction with the information, rather than the level of information itself, that correlates specifically with peri-operative anxiety levels. ${ }^{19}$ Perhaps it is this desire for complete clarity and understanding surrounding the surgical process that makes unfamiliar technical terms more alarming to women? 
While technical discussion and requests for equipment are a necessary part of the theatre environment, background conversation disrupts both communication and concentration among theatre staff. ${ }^{29}$ Noise in the operating room elicits stress related changes in the autonomic nervous system, which reduces performance among staff members and increases anxiety in conscious patients. ${ }^{30,31}$ It was therefore interesting to find that in our own cohort, general background noise was not reported to be stressful. Indeed, some patients reported that overhearing casual conversations among staff members was actually comforting.

There is some support for this finding in a study of patients undergoing hip replacement under regional anaesthesia. ${ }^{32}$ Although the noise of surgical drills was distressing, comforting words from nursing staff were a positive distraction. While the casual conversations to which our patients are exposed are not intended directly to be words of comfort, they may provide a welcome diversion from the technical discussions between staff members.

The effect of music on surgical patients has been studied widely ${ }^{33}$ and there is some evidence of a positive effect on both anxiety and blood pressure, particularly if the patient is able to select his or her own music. ${ }^{54,55}$ In our own study, over $90 \%$ of patients ranked music as 1 (causing no anxiety) and $16 \%$ of these would have liked to have the option of providing their own music. It has been suggested that it is the occupation of neurotransmitters via auditory stimulation by music that promotes this positive response, causing diversion of ill feeling, be that anxiety or pain. ${ }^{56}$ It is interesting to consider whether the mundane and casual conversations that our patients found comforting could be operating via a similar mechanism.

\section{Conclusions}

Overall anxiety levels among patients attending for local anaesthetic plastic surgery procedures were low and, for the most part, predictable in that they related to physical discomfort. However, this study identified that commonly used surgical terms, not specific to plastic surgery, can be an additional cause of distress. While it is widely recognised that an important relationship exists between patient anxiety and adequacy of communication with medical personel, ${ }^{16}$ the specific impact of technical terminology used in theatre has not yet been explored.

Our results suggest that some technical terms are more threatening than others with the same meaning. This raises the question of whether we should consider substituting one term for another in situations in which it is possible to do so. Further prospective study of the use of alternative words and how these impact on both patient anxiety and adequacy of communication between theatre staff is now needed to investigate this unexplored aspect of the awake surgery experience.

\section{References}

1. Wiles MD, Nathanson MH. Local anaesthetics and adjuvants - future developments. Anaesthesia 2010; 65 Suppl 1: 22-37.

2. Nordin P, Zetterström H, Gunnarsson U, Nilsson E. Local, regional, or general anaesthesia in groin hernia repair: multicentre randomised trial. Lancet 2003; 362: 853-858.

3. Schäffer J, Mehrmann M, Heymann-Schramm S et al. Perioperative anxiety and postoperative pain suppression in intraocular operations using general anesthesia and local anesthesia. Anaesthetist 1988; 37: 19-23.

4. Callesen T. Inguinal hernia repair: anaesthesia, pain and convalescence. Dan Med Bull 2003; 50: 203-218.

5. Wipfli M, Birkhäuser $\mathrm{F}$, Luyet $\mathrm{C}$ et al. Ultrasound-guided spermatic cord block for scrotal surgery. Br J Anaesth 2010; 106: 255-259.

6. Sessler DI. Does regional analgesia reduce the risk of cancer recurrence? A hypothesis. Eur J Cancer Prev 2008; 17: 269-272.

7. Hamerschmidt R, Mocellin M, Gasperin AC et al. Local anesthesia for cochlear implant surgery: a possible alternative. Braz J Otorhinolaryngol 2010; 76: 561-564.

8. Johnston M. Anxiety in surgical patients. Psychol Med 1980; 10: 145-152.

9. Wallace LM. Psychological preparation as a method of reducing the stress of surgery. J Human Stress 1984; 10: 62-77.

10. Maranets I, Kain ZN. Preoperative anxiety and intraoperative anesthetic reqirements. Anesth Analg 1999; 89: 1,346-1,351.

11. Williams JG, Jones JR. Psychophysiological responses to anesthesia and operation. JAMA 1968; 203: 415-417.

12. Tomlinson PJ, Field J. Warm or refrigerated local anaesthetic for open carpal tunnel release: a single blind randomized controlled study. J Hand Surg Eur Vol 2010; 35: 232-233.

13. Watts AC, McEachan J. The use of a fine-gauge needle to reduce pain in open carpal tunnel decompression: a randomized controlled trial. J Hand Surg $\mathrm{Br}$ 2005; 30: 615-617.

14. Nijkamp MD, Ruiter RA, Roeling $M$ et al. Factors related to fear in patients undergoing cataract surgery: a qualitative study focusing on factors associated with fear and reassurance among patients who need to undergo cataract surgery. Patient Educ Couns 2002; 47: 265-272.

15. Mitchell M. Conscious surgery: influence of the environment on patient anxiety. J Adv Nurs 2008; 64: 261-271.

16. Mokashi A, Leatherbarrow B, Kincey J et al. Patient communication during cataract surgery. Eye (Lond) 2004; 18: 147-151.

17. Brown SM. Quantitative measurement of anxiety in patients undergoing surgery for renal calculus disease. J Adv Nurs; 15: 962-970.

18. Bellani ML. Psychological aspects in day-case surgery. Int J Surg 2008; 6: S44-S46.

19. Williams OA. Patient knowledge of operative care. J R Soc Med 1993; 86 : 328-331.

20. Hansen $E$, Bejenke $C$. Negative and positive suggestions in anaesthesia: improved communication with anxious surgical patients. Anaesthesist 2010; 59: 199-202, 204-206, 208-209.

21. Munafó MR, Stevenson J. Selective processing of threat-related cues in day surgery patients and prediction of post-operative pain. Br J Health Psychol 2003; 8: 439-449.

22. Haugen AS, Eide GE, Olsen MV et al. Anxiety in the operating theatre: a study of frequency and environmental impact in patients having local, plexus or regional anaesthesia. J Clin Nurs 2009; 18: 2,301-2,310.

23. Jakobsen VH, Fagermoen MS. Environmental factors in the operating theatre and their impact on patients' preoperative anxiety. Tidsskrift For Sykepleieforskning 2005; 7: 4-17.

24. Gajraj NM, Sharma SK, Souter AJ et al. A survey of obstetric patients who refuse regional anaesthesia. Anaesthesia 1995; 50: 740-741.

25. Trento M, Tomelini M, Lattanzio R et al. Perception of, and anxiety levels induced by, laser treatment in patients with sight-threatening diabetic retinopathy. A multicentre study. Diabet Med 2006; 23: 1,106-1,109.

26. Birch BR, Chakraborty R, Miller RA. Anxiety in patients undergoing local anaesthetic day-case cystoscopy. Journal of One-Day Surgery 1993; 3: 15-17.

27. Khan FA, Nazir S. Assessment of pre operative anxiety in patients for elective surgery. J Anesth Clin Pharmacology 2007; 23: 259-262.

28. Keulers BJ, Scheltinga MR, Houterman $S$ et al. Surgeons underestimate their patients' desire for preoperative information. World J Surg 2008; 32: 964-970.

29. Hodge B, Thompson JF. Noise pollution in the operating theatre. Lancet 1990; 335: 891-894. 
30. Shankar N, Malhotra KL, Ahuja S, Tandon OP. Noise pollution: a study of noise levels in the operation theatres of a general hospital during various surgical procedures. J Indian Med Assoc 2001; 99: 244, 246-247.

31. Liu EH, Tan S. Patients' perception of sound levels in the surgical suite. J Clin Anesth 2000; 12: 298-302.

32. Hankela S, Kiikkala I. Intraoperative nursing care as experienced by surgical patients. AORN J 1996; 63: 435-442.

33. Nilsson $\mathrm{U}$. The anxiety- and pain-reducing effects of music interventions: a systematic review. AORN J 2008; 87: 780-807.

34. Yung PM, Chui-Kam S, French P, Chan TM. A controlled trial of music and pre-operative anxiety in Chinese men undergoing transurethral resection of the prostate. J Adv Nurs 2002; 39: 352-359.

35. Lee $D$, Henderson A, Shum $D$. The effect of music on preprocedure anxiety in Hong Kong Chinese day patients. J Clin Nurs 2004; 13: 297-303.

36. Cooke M, Chaboyer W, Schluter P, Hiratos M. The effect of music on preoperative anxiety in day surgery. J Adv Nurs 2005; 52: 47-55.

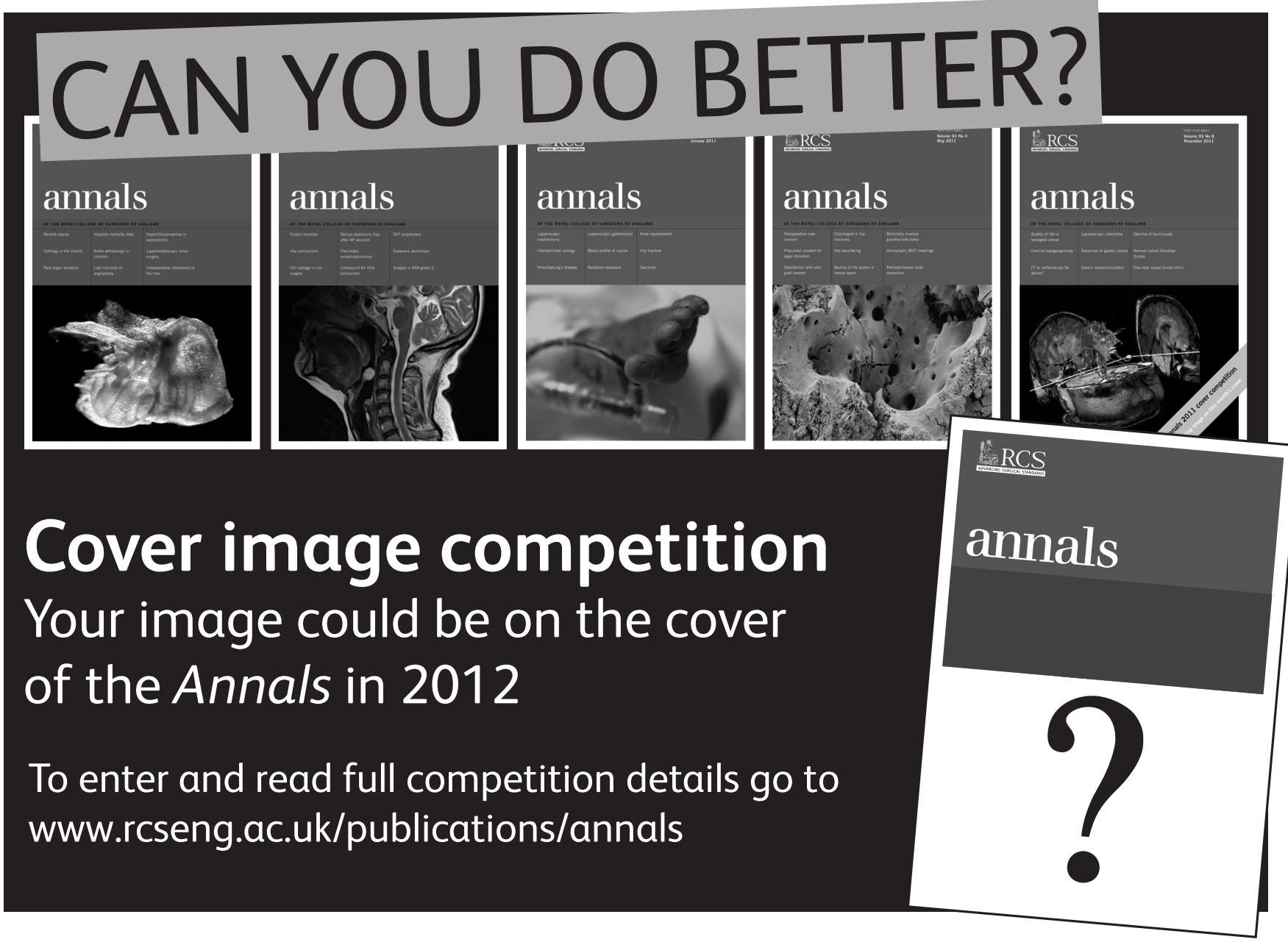

